\title{
Politik und Religion in der aktuellen politischen Wissenschaft. Mehr als politische Gewalt im Namen Gottes
}

\section{Einleitung: Religion und Politik in globalen Öffentlichkeiten}

Der 11. August 2010 war politisch ein normaler, ganz und gar unauffälliger Tag. Eine führende deutsche Tageszeitung informierte ihre Leser darüber, dass die Koalitionsverhandlungen zwischen Liberalen, Christdemokraten und dem »kämpferischen Islamkritiker Wilders « in den Niederlanden auch daran scheitern, dass die ersten beiden Parteien »den Islam als Religion [bezeichnen/ A.L.], Wilders hingegen sieht in ihm nur eine politische Ideologie«. In Italien teile Umberto Bossi, Chef der italienischen Lega Nord, Wilders Ressentiments »gegen Ausländer, insbesondere muslimische. $\aleph^{1}$ Im Libanon steige die Gefahr eines neuerlichen bewaffneten Konflikts mit weitreichenden regionalen Erschütterungen, weil die »Hizbullah, Iran, Syrien und die Hamas ihre Zusammenarbeit verstärkt haben. $\ll^{2}$ In Afghanistan stehe die Frage im Raum, ob sich Frieden nur dann herstellen lasse, wenn die Regierung in Kabul die Macht mit »den Oberislamisten Omar und Hekmatyar, an deren Händen das Blut Hunderttausender klebt « ${ }^{3}$, teile. Aus dem Iran wird berichtet, dass sieben führende Bahai, fünf Männer und zwei Frauen, in einem politischen Gerichtsverfahren aufgrund ihres Glaubens zu 20 Jahren Haft verurteilt worden sind. ${ }^{4}$ Und in Polen wühlt der Streit um den Verbleib des vor dem Präsidentenpalast errichteten, vier Meter hohen Gedenkkreuzes für den tödlich verunglückten Präsidenten Kaczynski die Öffentlichkeit auf. Mehrere tausend Menschen forderten in Sprechchören »die Beachtung des Prinzips der Trennung von Staat und Kirche. Anhänger und Gegner des Kreuzes beschimpften sich gegenseitig. « ${ }^{5}$

Dieser 11. August 2010 macht schlaglichtartig deutlich, wie nachhaltig und tief Religion in den politischen Alltag der Gegenwart eingedrungen ist. Die Zeichen mehren sich, dass die Zeiten vorbei sind, in denen man in der Politikwissenschaft erst welthistorische Vorgänge in Erinnerung rufen musste, um Aufmerksamkeit für den Zusammenhang für Politik und Religion in der Gegenwart zu finden. In der Tat sind die vielen global wahrgenommenen Ereignisse wie die Terroranschläge des 11. September, der Irakkrieg, reli-

1 Michael Stabenow, »Belgisch-niederländisches Menetekel« in: Frankfurter Allgemeine Zeitung (FAZ) vom 11.8.2010, 1.

2 Hans-Christian Rößler, »Gespannte Ruhe im Libanon« in: FAZ vom 11.8.2010, 2.

3 K. F., »Über Leichen« in: FAZ vom 11.8.2010, 8.

4 »Führende Bahai in Iran verurteilt« in: FAZ vom 11.8.2010, 5.

5 Hans-Christian Rößler, »Gespannte Ruhe im Libanon« in: FAZ vom 11.8.2010, 2. 
giös motivierte Selbstmordattentate in London und Madrid oder die politischen Entscheidungen zu Kopftuch, Burka, Moschee- und Minarettbau quer durch Europa Spitzenphänomene einer zunehmenden politischen Bedeutung von Religion im 21. Jahrhundert.

Die angeführten Beispiele zeigen zugleich, wie vielgestaltig das Thema Politik und Religion ist. Bereits auf der Ebene der Phänomene ist das hier zu behandelnde Thema so zerklüftet, dass es schwer fällt, die Orientierung zu behalten. Und diese Orientierung wird nicht gerade gefördert, wenn man den Blick hoffnungsvoll auf die Forschungen der Politikwissenschaft richtet. Und das aus doppeltem Grund.

Erstens hat die Politikwissenschaft in Deutschland, aber auch in anderen Ländern Religion lange Zeit bestenfalls als randständiges Thema ihres Faches angesehen. ${ }^{6}$ Und zweitens ist dieses alte Desinteresse gleichsam über Nacht verschwunden. Seit Mitte der 1990er Jahre ist die wissenschaftliche Literatur zum Thema »Politik und Religion« ebenso sprunghaft wie unsystematisch angewachsen.

Im Folgenden wird der Versuch unternommen, Schneisen durch diese jüngere politikwissenschaftliche Forschung zu legen, wobei der Fokus auf der deutsch- und englischsprachigen Forschung liegt. Dabei ist Vollständigkeit weder zu erreichen, noch erstrebt. ${ }^{7}$ Vielmehr möchte der vorliegende Beitrag eine Strukturierung, eine problemorientierte Systematik des breiten Forschungsfelds »Politik und Religion « vorschlagen. Ziel ist es, jene grundlegenden Problemzusammenhänge herauszuarbeiten, in denen das The$\mathrm{ma}$ »Religion « im Rahmen der konzeptionellen Grundlagen der Politikwissenschaft zu verorten ist. Aus meiner Sicht drängen sich dazu vier zentrale Problemkreise auf, die in der gebotenen gedanklichen Verdichtung im Folgenden vorgestellt werden sollen.

Diese Problemkreise sind:

- Religion und politische Gewalt: »Fundamentalismus«? (Abschnitt 2)

- Religion und politische Herrschaft: Staat-Kirche, Religionspolitik, Religionsfreiheit (Abschnitt 3)

6 Vgl. Ted Gerard Jelen / Clyde Wilcox, (Hg.), Religion and Politics in Comparative Perspective. The One, the Few, and the Many, Cambridge 2002; Kenneth D. Wald / Clyde Wilcox, »Getting Religion: Has Political Science Rediscovered the Faith Factor? « in: American Journal of Political Science 100 (2006) 523-529; Antonius Liedhegener, »Religion in der vergleichenden Politikwissenschaft. Begriffe - Konzepte - Forschungsfelder « in: Mathias Hildebrandt / Manfred Brocker (Hg.), Der Begriff der Religion (= Politik und Religion) Wiesbaden 2008, 179-196. Zu Recht hat man in der deutschen Politikwissenschaft lange Zeit von Religion als einer »terra incognita sprechen können. Vgl. Dieter Oberndörfer / Karl Schmitt, »Vorwort « in: dies. (Hg.), Kirche und Demokratie (= Sammlung Schöningh zur Geschichte und Gegenwart), Paderborn 1983, 7-9.

7 Eine erste Orientierung zur angelsächsischen Forschung liefert Jeffrey Haynes (Hg.), Routledge Handbook of Religion and Politics (= Routledge International International Handbooks) New York, NY 2009. Zahlreiche ältere englischsprachige Aufsätze versammelt John T. S. Madeley (Hg.), Religion and Politics (= The International Library of Politics and Comparative Government) Burlington, VT 2003; Aktuelle Forschungsüberblicke für die wichtigsten Teildisziplinen der Politikwissenschaft finden sich in Antonius Liedhegener / Andreas Tunger-Zanetti / Stephan Wirz (Hg.), Religion - Wirtschaft - Politik. Forschungszugänge zu einem aktuellen transdisziplinären Feld (= Religion - Wirtschaft - Politik, Bd. 1), Baden-Baden - Zürich 2011. 
- Religion und politische Entscheidungsprozesse: Macht und Einfluss kollektiver Akteure (Abschnitt 4), und

- Religion und Zivilgesellschaft: die Integration pluraler Gesellschaften (Abschnitt 5). Vorausgeschickt werden Überlegungen zur politikwissenschaftlichen Relevanz einiger grundlegender Begriffe und Konzepte, die die wissenschaftlichen Debatten zum Verhältnis von Religion und Moderne prägen (Abschnitt 1). Am Schluss des Beitrags stehen Überlegungen zu möglichen Konsequenzen der vorgestellten politikwissenschaftlichen Forschungen für eine politisch-praktische Gestaltung des Verhältnisses von Politik und Religion im Rahmen demokratischer Verfassungsstaaten.

\section{Religion in der Moderne: grundlegende Begriffe und Konzepte}

Am Anfang einer jeden wissenschaftlichen Beschäftigung mit dem Problemzusammenhang von Religion und Politik steht die Frage nach einem adäquaten Religionsbegriff. ${ }^{8}$ Dazu ist zunächst und einmal mehr auf die zentrale Bedeutung des Säkularisierungsparadigmas für die Wahrnehmung von Religion in den Geistes- und Sozialwissenschaften hinzuweisen. Die Annahme des mit der funktionalen Differenzierung moderner Gesellschaften und der Verbreitung rationaler Deutungs- und Handlungsmuster notwendig einhergehenden Niedergangs von Religion und Religiosität gehörte bis vor Kurzem weithin zum festen, nicht ernsthaft zu bezweifelnden Wissenskanon. Das trifft mehr noch für die angelsächsische als für die deutsche Forschung zu. ${ }^{9}$

Zwar erschloss Thomas Luckmann mit der These der »unsichtbaren Religion « ${ }^{10}$ der Religionssoziologie schon Ende der 1960er-Jahre einen neuen Deutungshorizont. Luckmann definiert Religion nicht mehr wie üblich als einen auf einen transzendenten Sinnoder Seinsgrund bezogenen gesellschaftlichen Teilbereich, sondern als ein individuelles Grundbedürfnis nach Sinn auch und gerade in der Moderne. Dieses Sinnbedürfnis kann nach Luckmann in mannigfaltigsten Formen erfüllt werden: in der Kunst, der Musik oder in Konsum und Popularkultur - die Moderne eröffne ein schier unbegrenztes »Warenlager 'letzter' Bedeutungen ${ }^{11}$ Seit Luckmann stehen sich in der Literatur substantielle

8 Vgl. Mathias Hildebrandt / Manfred Brocker (Hg.), Der Begriff der Religion (= Politik und Religion) Wiesbaden 2006; Karsten Lehmann, »Interdependenzen zwischen Religionsgemeinschaften und internationaler Politik. Religionswissenschaftliche Anmerkungen zu politikwissenschaftlichen Religionskonzeptionen « in: Zeitschrift für Internationale Beziebungen 17 (2010), 75-99.

9 Vgl. zu den Grundproblemen immer noch Karel Dobbelaere, »Secularization: A Multidimensional Concept « in: Current Sociology 29(1981), 1-216. Zusammenfassend Gert Pickel, Religionssoziologie: Eine Einfübrung in zentrale Themenbereiche, Wiesbaden 2011. Für die Rezeption des Paradigmas in der Politikwissenschaft vgl. derzeit vor allem Steve Bruce, Politics and Religion, Cambridge 2003; Steve Bruce, »Secularisation and Politics « in: Jeffrey Haynes (Hg.), Routledge Handbook of Religion and Politics (= Routledge International Handbooks) New York, NY 2009, 145-158.

$10 \mathrm{Vgl}$. Thomas Luckmann, Die unsichtbare Religion. Mit einem Vorwort von Hubert Knoblauch, Frankfurt/M. 1991.

11 Ebd., 145. 
und formale Religionsdefinitionen unversöhnlich gegenüber. Die Einführung des formalen Luckmann'schen Religionsbegriffs steigerte die Attraktivität des Gegenstandes »Religion « für die Politikwissenschaft nicht. Welche relevanten Wirkungen sollten auch von einer unsichtbaren, diffusen Religion auf politische Willensbildungs- und Entscheidungsprozesse ausgehen?

Jenseits der politikwissenschaftlichen Teildisziplin »Ideengeschichte/ politische Theorie « ${ }^{12}$ erwachte das politikwissenschaftliche Interesse an Religion erst wieder, als vor allem in den USA die »Rückkehr der Religionen « ausgerufen wurde. Faktisch spielte der weltweit erstarkende Islam dabei eine zentrale Rolle. Für die theoretische Debatte war dies aber zunächst von eher untergeordneter Bedeutung. Geradezu furiose Angriffe auf die liebgewonnene Säkularisierungsthese kamen von überraschender Seite, nämlich von der rational-choice-Theorie. Nach den Vertretern der sog. »Markttheorie der Religion « sind für die tatsächliche Vitalität von Religion kein imaginärer Geschichtstrend, sondern die Art und der Umfang des religiösen Angebotes entscheidend. Je mehr religiöse Anbieter und je kompetitiver der religiöse Markt, desto besser werde die Nachfrage nach Religion bedient. Das erkläre die enorme religiöse Vitalität der USA. »After nearly three centuries of utterly failed prophesies [...], it seems time to carry the secularization doctrine to the graveyard of failed theories, and there to whisper 'requiescat in pace'.. ${ }^{13}$ Bekanntlich leben Totgesagte länger; die Empirie spricht gegen dieses Begräbnis erster Klasse. $^{14}$

Aus sozialwissenschaftlicher und vor allem politikwissenschaftlicher Sicht einen wirklichen Schritt nach vorn lieferte 1994 das Buch von José Casanova »Public Religions «. ${ }^{15}$ Anhand von fünf Fallstudien konnte Casanova die Annahme des umfassenden Bedeutungsverlusts von Religion in modernen Gesellschaften empirisch zurückweisen. Dabei legte er einen substantiellen Religionsbegriff zugrunde. Konzeptionell verabschiedete er das Säkularisierungsschema damals aber nicht, sondern führte eine dreifache Unterscheidung ein, die sich bewährt und vielfach durchgesetzt hat. Säkularisierung betrifft danach erstens die Trennung von Staat und Religion, zweitens die Rolle der Religion in

12 Vgl. Hans Maier (Hg.), »Totalitarismus« und »Politische Religionen «. Konzepte des Diktaturvergleichs (= Politik- und Kommunikationswissenschaftliche Veröffentlichungen der GörresGesellschaft, Bd.16) Paderborn u.a. 1996; Manfred Walther (Hg.), Religion und Politik. Zu Theorie und Praxis des theologisch-politischen Komplexes (= Schriftenreihe der Sektion Politische Theorien und Ideengeschichte in der DVPW, Bd.5), Baden-Baden 2004; Aktueller Forschungsüberblick in Armin Pfahl-Traughber, » Politische Religion< und `Zivilreligion< - Politische Theorie« in: Antonius Liedhegener / Andreas Tunger-Zanetti/ Stephan Wirz (Hg.), Religion - Wirtschaft - Politik. Forschungszugänge zu einem aktuellen transdisziplinären Feld (= Religion - Wirtschaft - Politik, Bd.1) Baden-Baden - Zürich 2011, 223-240.

13 Rodney Stark / Roger Finke, Acts of Faith, Berkely, CA 2000, 79. Vgl. auch Rodney Stark, »Secularization, R.I.P.« in: Sociology of Religion 60 (1999), H.3, 249-265.

14 Vgl. die Befunde in Pippa Norris / Ronald Inglehart, Sacred and Secular: Religion and Politics Worldwide (= Cambridge Studies in Social Theory, Religion, and Politics), Cambridge 2004; Detlef Pollack, Säkularisierung - ein moderner Mythos?, Tübingen 2003; Detlef Pollack, Rückkebr des Religiösen? (= Studien zum religiösen Wandel in Deutschland und Europa, Bd.2) Tübingen 2009.

15 Vgl. José Casanova, Public Religions in the Modern World, Chicago - London 1994.

ZfP 58. Jg. 2/2011 
der Zivilgesellschaft und drittens ihre Verflechtung mit bzw. ihren Einfluss auf das politische Entscheidungszentrum. Casanova wendete diese Unterscheidung auch normativ: Die Trennung sei unhintergehbar, der Ausschluss von Religion aus politischen Entscheidungsprozessen für moderne Demokratien eine Grundvoraussetzung. Nicht zwingend seien jedoch der Niedergang von Religion allgemein und - das war die besondere Pointe - der Ausschluss religiöser Akteure von der politischen Debatte demokratischer Öffentlichkeiten. Nach Casanovas ursprünglichem Konzept der 'public religion' ist daher die Zivilgesellschaft der (einzig) legitime Ort der Religion. ${ }^{16}$

Casanovas Buch wies so einen Weg aus den theorieorientierten religionssoziologischen Debatten in die empirische Politikforschung. Im Vergleich zur Soziologie, aber auch zur jüngeren Religionswissenschaft, wo die Dinge strittig sind, dominiert in der Politikwissenschaft dementsprechend heute ganz überwiegend ein substantielles Religionsverständnis. ${ }^{17}$ Speziell in der Vergleichenden Politikwissenschaft deckt sich Religion de facto meist mit den großen Weltreligionen, ihren Varianten und kollektiven Vertretern. ${ }^{18}$ Anders gesagt gilt für die politische Wissenschaft: Religion im Singular gibt es empirisch nicht.

Angesichts dessen kann die Politikwissenschaft die jüngeren religionssoziologischen Aufgeregtheiten um das Für und Wider von alter Wiederkehr und neuer Säkularisierung m.E. mit einer gewissen Gelassenheit verfolgen. ${ }^{19}$ Unbedingt hinhören sollte die Poli-

16 Vgl. ebd., 211-234; ders., »What Is a Public Religion? « in: Hugh Heclo / Wilfried M. McClay (Hg.), Religion Returns to the Public Square. Faith and Policy in America, Washington DC 2003, 111-139. - In seinen neueren, m. E. politiktheoretisch weniger überzeugenden Arbeiten rückt Casanova von seinem ursprünglichen Konzept der spublic religion< deutlich ab. José Casanova, »Public Religions Revisited « in: Hermann-Josef Grosse Kracht / Christian Spiess (Hg.), Christentum und Solidarität. Bestandsaufnabmen zu Sozialethik und Religionssoziologie, Festschrift für Karl Gabriel, Paderborn 2008, 313-338; José Casanova, Europas Angst vor der Religion, Berlin 2009. Das legitime und vernünftige Anliegen, die Hürden zwischen Demokratie und den Traditionen der verschiedensten Religionen dieser Welt nicht zu hoch zu setzen, hat ihn bewogen, vom Gedanken der Trennung von Staat und Religion und dessen Fundierung in der Religionsfreiheit abzurücken. An die Stelle universaler, globaler und daher staatlich zu wahrender und zu schützender Menschenrechte tritt in seinen jüngsten Arbeiten ein Kulturalismus der »Anerkennung der unabänderlichen Pluralität der Universalismen«, so Casanova, ebd., 114. Casanova plädiert dafür, der Religionsfreiheit als einem historisch kontingenten Ergebnis der europäisch-westlichen Moderne die »Anerkennung der kollektiven Rechte von Völkern « (ebd., 112) und ihrer kulturellen und religiösen Traditionen entgegenzusetzen und folgert daraus ein »allgemeines Prinzip der reziproken Rechte und Pflichten aller Völker der Welt [...], gegenseitig die Traditionen und Kulturen des Anderen zu respektieren«; ebd., 113.

17 In der Disziplin "zeigt sich insgesamt eine gewisse Konvergenz [des Religionsbegriffs]: Transzendenzbezug, kollektive Sinnstiftung und Institutionalisierung sind die zentralen Charakteristika «, Liedhegener, Religion, 5.

18 Wobei gleich hinzuzufügen ist, dass die große Varianz innerhalb der religiösen Traditionen je nach Zeit, Region und spezifischer Ausprägung stets mitzudenken ist; Jelen / Wilcox (Hg.), Religion, 4 und 7.

19 Vgl. zur Unübersichtlichkeit der Lage etwa Thomas M. Schmidt, »Die Wiederkehr der Säkularisierung. Postsäkulare Gesellschaft im postreligiösen Zeitalter« in: Fuge. Journal für Religion E Moderne 1 (2007), 109-122. 
tikwissenschaft aber, wenn Jürgen Habermas und Joseph Ratzinger über die Zuordnung von Vernunft und Religion in der Moderne diskutieren. ${ }^{20}$ Habermas, von den jüngeren Entwicklungen etwa der Gen-Technik zutiefst beunruhigt, stellt die Frage nach der Bedeutung von Religion für die vorpolitischen Grundlagen demokratischer Ordnung in drängender Weise neu und spricht von der rettenden Wirkung der Religion. Ratzinger interpretiert die Gewaltgeschichte Europas und die Gefahren der technologischen Entwicklung als Brüchigkeit sowohl der Religion als auch der praktischen Vernunft. ${ }^{21} \mathrm{Im}$ Hinblick auf die Gegenwart wirft er die Frage auf: »Wenn Terrorismus auch durch religiösen Fanatismus gespeist wird - und er wird es -, ist dann Religion eine beilende und rettende, oder nicht eher eine archaische und gefäbrliche Macht, die falsche Universalismen aufbaut und dadurch zu Intoleranz und Terror verleitet«?22

\section{Religion und politische Gewalt: »Fundamentalismus«?}

Die jüngere Debatte um den Zusammenhang von Religion und Gewalt ist unzweifelhaft durch die Arbeiten Samuel Huntingtons geprägt worden. Sowohl der berühmte Aufsatz »Clash of Civilizations? « - mit Fragezeichen - als auch das nachgeschobene gleichnamige Buch - nun ohne Fragezeichen - haben breite Aufmerksamkeit in der Öffentlichkeit und ebenso breite Ablehnung in der Fachwissenschaft gefunden. ${ }^{23}$ Huntingtons Thesen trugen »maßgeblich zur Wahrnehmung der Religion als eines zentralen Konflikt auslösenden Momentes der aktuellen Weltpolitik - und damit zur exponentiell wachsenden Zahl von Publikationen zur 'Gefährlichkeit der Religionen' - bei.« ${ }^{24}$ Insbesondere der Islam gilt Huntington, weil prinzipiell nicht demokratisierbar, als Herd kriegerischer Konflikte.

$\mathrm{Ob}$ und in welchem Umfang Religionen tatsächlich für politische Gewalt im Sinne von Terror und kriegerischen Auseinandersetzungen ursächlich sind, ist in der Politikwissenschaft heftig umstritten. Insbesondere unter Vertretern der Teildisziplin der Internationalen Beziehungen herrscht die Ansicht vor, dass »religiöse Elemente [zwar vielfach...] mehr oder weniger ausgeprägt vorhanden sind, dass sie aber gegenüber den machtpolitischen und wirtschaftlichen Aspekten in der Regel eine eher sekundäre Be-

20 Vgl. Jürgen Habermas / Joseph Ratzinger, Dialektik der Säkularisierung. Über Vernunft und Religion, mit einem Vorwort von Florian Schuller, Freiburg - Basel - Wien 22005.

21 Christentum und Aufklärung seien daher wechselseitig aufeinander verwiesen. Vgl. ebd., 48 und 57-58.

22 Ebd., 46-47 (Hervorhebung im Original).

23 Samuel P. Huntington, »The Clash of Civilizations? « in: Foreign Affairs 72 (1993), H. 3, 22-49; ders., Kampf der Kulturen. Die Nengestaltung der Weltpolitik im 21. Jabrbundert, aus dem Amerikanischen von Holger Fliessbach, München - Wien 1996 (am. 1996: The Clash of Civilizations).

24 Manfred Brocker, »Einleitung: Friedensstiftende Religionen? Religion und die Deeskalation politischer Konflikte« in: Manfred Brocker / Mathias Hildebrandt (Hg.), Friedensstiftende Religionen? Religion und die Deeskalation politischer Konflikte (= Politik und Religion), Wiesbaden 2007, 9-25, hier 9 . 
deutung einnehmen. ${ }^{25}$ Religiösen Vorstellungen wird im Einzelfall eine konfliktverschärfende Wirkung zugeschrieben. Die Lösung des Zusammenhangs von Religion und Gewalt besteht aus der Perspektive des Realismus dann »kurz gesagt, in einer gewissen Übertragung von Grundprinzipien des > Westfälischen Friedens < [...]: Koexistenz auf der Grundlage der Anerkennung der - politisch wie religiös fundierten - Unterschiede. « 26

Es erscheint aber voreilig, Religionen jegliche Akteursqualität in kriegerischen Konflikten abzusprechen. In der deutschsprachigen Literatur wird diese These vor allem von Andreas Hasenclever und Volker Rittberger vertreten. ${ }^{27}$ Religionsgemeinschaften und religiöse Vorstellungen sind danach allein instrumentelle Ressourcen, die mehr oder weniger skrupellose politische Eliten in gewaltsamen Konflikten gezielt und kalkuliert einsetzen; d.h. Religion wird ausschließlich als abhängige Variable der Machtpolitik gesehen.

Den wohl wichtigsten empirischen Beitrag zum Zusammenhang von Religion und Gewalt hat das von mehreren amerikanischen Universitäten getragene Fundamentalismus-Projekt geliefert. ${ }^{28}$ Dieses von der renommierten American Academy of Arts and Science geförderte Großvorhaben ist auch in methodischer Hinsicht beachtenswert. Entgegen dem Trend einer theoriegeleiteten, auf quantifizierbare Kausalitäten gerichteten empirischen Forschung hat man sich hier für ein induktives, hermeneutisches Vorgehen entschieden. Der gemeinsame Ausgangspunkt bestand darin, dass der Begriff Fundamentalismus aus seinem amerikanischen Entstehungskontext gelöst und als Oberbegriff für eine spezifische Ausprägung von Religionen im Gegenüber zur Moderne verwandt wurde. » Fundamentalism [...] refers to a discernible pattern of religious militance by which self-styled true beliefers attempt to arrest the erosion of religious identity, fortify the borders of the religious community, and create viable alternatives to secular institutions and behaviors. «29

Die Schwierigkeit dieses Ansatzes liegt in der Formulierung »religiöse Militanz«. Warum? Diese Formulierung überwölbt oder verdeckt die nach der Definition offene

25 Helmut Hubel, »Wie viel Religion ist in den Konflikten des Vorderen Orients? « in: Mathias Hildebrandt / Manfred Brocker (Hg.), Unfriedliche Religionen? Das politische Gewalt- und Konfliktpotential von Religionen (= Politik und Religion), Wiesbaden 2005, 179-190, hier 190.

26 Ebd.

27 Vgl. Andreas Hasenclever / Volker Rittberger, The Impact of Faith: Does Religion Make a Difference in Political Conflict? (= Tübinger Arbeitspapiere zur internationalen Politik und Friedensforschung, Nr. 35), Tübingen 1999; Andreas Hasenclever, »Merkmale gewaltresistenter Glaubensgemeinschaften - Überlegungen zum Schutz religiöser Überlieferung vor politischer Vereinnahmung « in: Mathias Hildebrandt / Manfred Brocker (Hg.), Unfriedliche Religionen? Das politische Gewalt- und Konfliktpotential von Religionen (= Politik und Religion) Wiesbaden 2005, 179-201; Andreas Hasenclever, Andreas / Alexander de Juan, »Religionen in Konflikten. Eine Herausforderung für die Friedenspolitik « in: APuZ (2007) B6, 10-16.

28 Vgl. dazu vor allem Scott Appleby / Martin E. Marty (Hg.), Fundamentalism Observed (= The Fundamentalism Project, Bd.1), Chicago 1991; Scott Appleby / Martin E. Marty (Hg.), Fundamentalism and the State: Remaking Polities, Economies, and Militance (= The Fundamentalism Project, Bd.3), Chicago 1993; Gabriel A. Almond / R. Scott Appleby, / Emmanuel Sivan, Strong Religion. The Rise of Fundamentalism around the World, Chicago - London 2003.

29 Ebd., 17. 
Frage, ob Fundamentalismus notwendig Gewalt mit einschließt. Die Autoren des Abschlussberichts verneinen dies, sprechen aber von einer »strong tendency ${ }^{30}$ zur Gewalt. ${ }^{31}$ Die Ursache dafür sehen sie in dem von fundamentalistischen Gruppierungen ausgehenden Machtanspruch nicht nur gegenüber ihren Mitgliedern, sondern auch gegenüber dem als feindlich angesehenen Staat.

Zwei besondere Leistungen bzw. Ergebnisse dieses Forschungsprojekts seien hervorgehoben: Erstens die Tatsache, dass der deskriptive Zugang die religiösen Inhalte und gedanklichen Konstrukte fundamentalistischer Gruppen offenlegt, d.h. Religion selbst wird Gegenstand der Untersuchung. Es zeigt sich, dass fundamentalistische Gruppen ein sehr voluntaristisches Verhältnis zu den religiösen Überlieferungen der eigenen Herkunftsreligion haben: Als gültig werden von fundamentalistischen Gruppen nur jene religiösen Aussagen vorgestellt, die dem Ziel der eigenen Behauptung und Durchsetzung in einer als feindlich aufgefassten Umwelt dienen. ${ }^{32}$

Und zweitens tritt die instrumentelle Rolle von Gewalt als Teil übergeordneter politischer Strategien religiös-fundamentalistischer Bewegungen hervor. ${ }^{33}$ Sie setzen Gewalttaten meist gezielt als operative oder strategische Gewalt zum Systemwechsel oder als expressive Gewalt ein, die Handlungsfähigkeit demonstrieren soll. ${ }^{34}$

Unbeschadet der Bedeutung der vorgelegten Ergebnisse erscheint die mit dem Stichwort »Fundamentalismus « automatisch verbundene Perspektive, Religion allein mit konfliktiven und gewalttätigen Situationen zu verknüpfen, fraglich. Diese Verkürzung mag sich angesichts der zeitgenössischen Erfahrungen und ihrer medialen Aufbereitung aufdrängen. Sie entspricht aber weder der Breite der politisch relevanten Phänomene, die mit Religionen in Zusammenhang stehen, noch dem Stand der Forschung, die nicht nur

30 Ebd.

31 Vgl. als Überblick zur Entwicklung in den verschiedenen Weltreligionen und -regionen jetzt Mark Juergensmeyer, Die Globalisierung religiöser Gewalt. Von christlichen Milizen bis al Quida, aus dem Englischen von Helmut Dierlamm und Thomas Pfeiffer, Hamburg 2009.

32 Vgl. Almond/ Appleby/ Sivan, Strong Religion (FN 28), 18-21.

33 Vgl. ebd., 234-237.

34 Erstere wird bewusst eingesetzt, um politische Ziele durch Gewalt zu erreichen. Prominentestes Beispiel war und ist die Revolution Khomeinis im Iran im Jahr 1979. Expressive, meist besonders grausame Gewalttaten richten sich gegen Einzelne oder kleinere Gruppen. Das Ziel expressiver Gewalt ist es, Handlungsfähigkeit zu demonstrieren und die Bereitschaft der Anhänger zum Kampf für die vermeintlich göttliche Sache öffentlich unter Beweis zu stellen. Fundamentalismus kann darüber hinaus fanatisierte Anhänger hervorbringen, die dann als Einzeltäter Anschläge oder politische Morde verüben. Die Ermordung des israelischen Ministerpräsidenten Rabin war beispielsweise das Werk eines jüdisch-orthodoxen Einzelgängers. Diese Art von begrenzter, im Einzelfall gleichwohl verheerender Gewalt lässt sich nicht unmittelbar organisierten fundamentalistischen Bewegungen zuschreiben. Ähnlich wird die spontane kollektive Gewalt von Menschenmassen eingeordnet, die sich etwa nach kleineren Zusammenstößen im Alltag entlädt oder an Begräbnisfeiern ermordeter Familienmitglieder entzündet. Fundamentalisten stacheln solche spontanen Gewaltorgien sehr wohl oftmals zusätzlich an, aber »riots and mob action would likely continue apace in the absence of fundamentalist manipulators.« Ebd., 235. 
immer öfter die friedensfördernden Potentiale von Religionen hervorhebt, ${ }^{35}$ sondern wie die folgenden drei Problemkreise zeigen - auch erheblich breitere Fragestellungen und entsprechende Forschungsergebnisse aufzuweisen hat.

\section{Religion und Herrschaft: Staat-Kirche, Religionspolitik, Religionsfreiheit}

Im Rahmen der Teildisziplin »Regierungslehre/Politische Systemlehre« ist das Verhältnis von Staat und Kirchen bzw. Religionsgemeinschaften das klassische Thema. Diese Sicht teilt die Politikwissenschaft vor allem mit der Rechtswissenschaft. Tatsächlich nehmen Forschungen zum Staat-Religion-Verhältnis in der gegenwärtigen Politikwissenschaft einen überraschend breiten Raum ein. Einen neuen Dreh hat diese Forschungsrichtung nämlich durch die Vergleichende Politikwissenschaft erhalten. Deren jüngste Beschäftigung mit dem Verhältnis von Staat und Religionen beruht nämlich auf der Annahme, dass unterschiedliche religionsrechtliche Arrangements Auskunft über die tatsächliche politische Bedeutung einzelner Religionen und Konfessionen in unterschiedlichen politischen Systemen und in deren verschiedenen Policy-Feldern geben könnten. Ziel ist es, das in einer abstrakten Betrachtung durchaus plausible Kontinuum zwischen einer theokratischen Herrschaft einerseits und einer cäsero-papistischen Herrschaftsform andererseits anhand weniger Kriterien so zu zergliedern, dass eine aussagekräftige Typologie der Zuordnung von Staat und Religionsgemeinschaften entsteht. Wichtige Vorschläge dazu stammen etwa von Marc Chaves und David Cann, Michael Minkenberg sowie Ronald Inglehart und Pippa Norris. ${ }^{36}$ Faktisch haben sich aber schon die historisch gewachsenen, unterschiedlichen Realisierungen der Trennung von Staat und Kirche in Europa, deren rechtliche Gestalt vor allem Gerhard Robbers untersucht hat, als äußerst

35 Vgl. mit weiterer Literatur dazu Ines-Jacqueline Werkner, »Krieg, politische Gewalt und Frieden - Religion und ihre Bedeutung in den Internationalen Beziehungen « in: Antonius Liedhegener / Andreas Tunger-Zanetti / Stephan Wirz (Hg.), Religion - Wirtschaft - Politik. Forschungszugänge zu einem aktuellen transdisziplinären Feld (= Religion - Wirtschaft - Politik, Bd.1) Baden-Baden - Zürich 2011, 305-324.

36 Vgl. Mark Chaves / David E. Cann, »Regulation, Pluralism, and Religious Market Structure. Explaining Religion's Vitality « in: Rationality and Society 4 (1992), 272-290; Michael Minkenberg, »Abtreibungsregime im Vergleich: Religiöse Einflüsse auf >Public Policy< in westlichen Demokratien « in: Manfred Brocker / Hartmut Behr / Mathias Hildebrandt (Hg.), Religion Staat - Politik. Zur Rolle der Religion in dernationalen und internationalen Politik (= Religion und Politik) Wiesbaden 2003, 127-147;

Michael Minkenberg, »Staat und Kirche in westlichen Demokratien « in: Michael Minkenberg / Ulrich Willems (Hg.), Politik und Religion (= PVS-Sonderheft, Bd.33), Wiesbaden 2003, 115-138; Pippa Norris / Ronald Inglehart, Sacred and Secular: Religion and Politics Worldwide (= Cambridge Studies in Social Theory, Religion, and Politics), Cambridge 2004. 
sperrig gegen solche Systematisierungsversuche erwiesen. ${ }^{37}$ Für alle Staaten dieser Welt scheint die Aufgabe denn auch kaum lösbar. Zwei Beispiele mögen das illustrieren.

Robert Barro und Rachel McCleary, in Harvard lehrende Vertreter der rationalchoice-Theorie, haben aus der genannten Not eine Tugend gemacht. Sie haben die auf den ersten Blick denkbar einfachste Frage gestellt: »Which Countries Have State Religions?«Anhand religionswissenschaftlicher Nachschlagewerke haben sie das Vorhandensein einer Staatsreligion Land für Land für die Stichjahre 1900, 1970 und 2000 geprüft und binär kodiert. Für die USA ergab die Prüfung »nein, nein, nein «, für Afghanistan »ja, ja, ja«, für Australien »nein, nein, nein«, und für Deutschland - »nein, nein, nein«. Jeder historisch Informierte dürfte spätestens jetzt verstört aufschrecken. Das Staat-Kirchen-Verhältnis im Kaiserreich und das der Bundesrepublik Deutschland unterscheiden sich fundamental. Einer solchen binären Kodierung kann angesichts der Komplexität des Gegenstandes m.E. keine inhaltliche Aussagekraft beigemessen werden.

Dem in Israel lehrenden, amerikanischen Politikwissenschaftler Jonathan Fox kommt das Verdienst zu, die Aufgabe einer differenzierten Typologisierung des Verhältnisses von Staat und Religionen für die Staaten der Welt empirisch in der bislang umfassendsten Art und Weise in Angriff genommen zu haben. Im Rahmen seines »Religion and State «Projekts hat Fox für 175 Länder jeweils über 60 Variablen insbesondere zu Religionsfreiheit, zum Religionsrecht und zur staatlichen Praxis gegenüber Religionen zusammengetragen. ${ }^{38}$ Der von ihm auf dieser Datenbasis gebildete Index, der »Government in Religion Involvement «-Index, kann Werte zwischen 0 und 100 annehmen. Null steht dabei für eine rechtlich strikte Trennung bei gleichzeitiger strikter Nichteinmischung in die religiöse Praxis der Bevölkerung. ${ }^{39}$ Eine vollständige Trennung kennt danach nur die USA. Weltweit greifen die allermeisten Regierungen mehr oder weniger stark in religiöse Belange ein. Bezogen auf die Rechtsbeziehungen in den untersuchten Ländern sind zwar

37 Vgl. Gerhard Robbers, »Status und Stellung von Religionsgemeinschaften in der Europäischen Union « in: Michael Minkenberg / Ulrich Willems (Hg.), Politik und Religion (= PVS-Sonderheft, Bd.33) Wiesbaden 2003, 139-163; Gerhard Robbers (Hg.), Staat und Kirche in der Europäischen Union, herausgegeben in Zusammenarbeit mit dem European Consortium for State and Church Research, 2. Aufl., Baden-Baden 2005; Karl Graf Ballestrem / Sergio Belardinelli / Thomas Cornides (Hg.), Kirche und Erziebung in Europa (= Politik und Religion) Wiesbaden 2005.

38 Vgl. Jonathan Fox, »World Separation of Religion and State Into the 21st Century« in: Comparative Political Studies 39 (2006), 537-569; ders., »Do Democracies Have Separation of Religion and State? « in: Canadian Journal for Political Science 40 (2007) H.1, 1-25; ders., A World Survey of Religion and the State (= Cambridge Studies in Social Theory, Religion and Politics) Cambridge 2008. - Überblick zur vergleichenden Politikwissenschaft jetzt in Gert Pickel, »Demokratie, Staat und Religionen. Vergleichende Politikwissenschaft « in: Antonius Liedhegener / Andreas Tunger-Zanetti / Stephan Wirz (Hg.), Religion - Wirtschaft - Politik. Forschungszugänge zu einem aktuellen transdisziplinären Feld (= Religion - Wirtschaft - Politik, Bd.1) Baden-Baden - Zürich 2011, 275-303.

39 Die grundlegende Definition von Trennung nach Fox lautet: »no government support for religion and no government interference in the religious practices of both the majority and the minority religions in the state«; Fox, World Separation (FN 38), 538. Zur Rezeption der Ergebnisse vgl. John Madeley, Religion and the State in: Jeffrey Haynes (Hg.), Routledge Handbook of Religion and Politics (= Routledge International Handbooks) New York, NY 2009, 174-191.

ZfP 58. Jg. 2/2011 
nur ganz wenige Staaten, nämlich 0,7 Prozent der erfassten Länder dezidiert religionsfeindlich (darunter aber das große China). In 21,7 Prozent der erfassten Staaten existiert dagegen offiziell eine Staatsreligion, in weiteren 21,1 Prozent der Fälle findet Fox eine inoffizielle Staatsreligion. ${ }^{40}$ Kritisch anzumerken ist, dass die verschiedenen von Fox entwickelten Skalen meist keine Trennschärfe zwischen kooperativen Trennungsmodellen in Demokratien und privilegierten Religionen in nichtdemokratischen Systemen herstellen. ${ }^{41}$

Vermutlich stößt die makro-quantitative komparative Forschung beim Thema Staat und Religionen aber auch an die Grenzen ihrer Erkenntnisleistung. Weitaus ergiebiger und politisch relevanter dürfte es auf Dauer sein, sich mit den Veränderungen des Verhältnisses von Staat und Religion in vergleichenden Fallstudien beschreibend und analysierend auseinanderzusetzen. Auf diesem methodischen Weg der qualitativ-komparativen Forschung hat vor allem Stephen Monsma herausgearbeitet, dass es unterhalb der immer noch weit verbreiteten, aber immer fragwürdiger werdenden typologischen Einordnung der verschiedenen Staaten in staatskirchliche Systeme, Trennungssysteme oder kooperative Systeme ${ }^{42}$ in den vergangenen Jahrzehnten zu einer erheblichen Angleichung der tatsächlichen rechtlichen Regelungen gekommen ist. Diesen Befund hat Monsma zu der generalisierenden These verdichtet, dass sich die Demokratien der Gegenwart in der Frage der rechtlichen und praktischen Ausgestaltung des Verhältnisses von Staat und Religionen auf einem Konvergenzkurs befinden. ${ }^{43}$ Vieles spricht dafür, dass sich dieser Zug zur Konvergenz der religionsrechtlichen Arrangements auch auf der Ebene der Europäischen Union, deren Verhältnis zur Religion erst jüngst zum Gegenstand politikwissenschaftlicher Forschung geworden ist, durchsetzen wird. ${ }^{44}$ Jedenfalls sind mit dem Vertrag von Lissabon unbeschadet der vorausgehenden Konflikte um den Bezug der Präambel auf die christliche Tradition Europas wesentliche Änderungen in Kraft getreten, die nicht nur die bestehenden kooperativen nationalstaatlichen Arrangements schützen (Art. 17 Abs. 1 des »Vertrags über die Arbeitsweise der Europäischen Union«),

40 Vgl. Fox, World Separation (FN 38), 552.

41 Pointiert zur Bedeutung dieses gewichtigen normativen Unterschieds auf der Basis von Daten zur Religionsfreiheit vgl. Wolfgang Merkel, »Religion, Fundamentalismus und Demokratie « in: Wolfgang Schluchter (Hg.), Fundamentalismus, Terrorismus, Krieg, Weilerswist 2003, 61-86.

42 Vgl. zu dieser kritischen Einschätzung auch Robbers, Status, 141: »Diese herkömmlichen Kategorien verlieren [...] zunehmend an juristischer Ordnungskraft, sie taugen eher als emotionale Erinnerungstopoi und verstellen den Blick auf faktische Entwicklungen und Problemlagen.«.

43 Vgl. Stephen V. Monsma / J. Christopher Soper, The Challenge of Pluralism. Church and State in Five Democracies (= Religious Forces in the Modern Political World), Lanham u.a. 1997; Stephen V. Monsma, "Staat und Kirche in den USA und der Bundesrepublik Deutschland: auf dem Weg zur Konvergenz? « in: Heinz-Dieter Meyer / Michael Minkenberg / Ilona Ostner (Red.), Religion und Politik. Zwischen Universalismus und Partikularismus (= Jahrbuch für Europa- und Nordamerika-Studien, Bd.2), Opladen 2000, 81-107. Vgl. gleichlautend auch Robbers, Status, 147-148.

44 Vgl. mit weiterer Literatur zum aktuellen Forschungsstand Antonius Liedhegener, »Mehr als Binnenmarkt und Laizismus? Die neue Religionspolitik der Europäischen Union « in: Martin Baumann / Frank Neubert (Hg.), Religionspolitik - Öffentlichkeit - Wissenschaft: Studien zur Neuformierung von Religion in der Gegenwart, Zürich 2011, 59-80. 
sondern zugleich für einen Übergang zu einem kooperativen Verhältnis der EU mit den Religionsgemeinschaften stehen. Dies kommt etwa im strukturierten Dialog der EU mit den Kirchen, Religionsgemeinschaften und weltanschaulichen Gemeinschaften zum Ausdruck (Art. 17 Abs. 3 AEU-Vertrag). Der Zugang der politikwissenschaftlichen Forschung über vergleichende Fallstudien macht zudem auch sichtbar, dass mit der Frage nach dem Staat-Religionen-Verhältnis im Recht und in der politischen Praxis immer auch normative Grundfragen des jeweiligen politischen Systems berührt werden. Das gilt auch für die Bundesrepublik Deutschland.

In der deutschen Politikwissenschaft gibt es in der Frage der Zukunftsfähigkeit der überkommenen kooperativen Trennung gegenwärtig einen Dissens. Kritiker wie Claus Leggewie, Volker von Prittwitz oder Ulrich Willems haben eine Totalrevision gefordert. ${ }^{45}$ Es gelte, die »religiös-christlich-großkirchliche Schlagseite ${ }^{46}$ der derzeitigen religionspolitischen Verfassung der Bundesrepublik zu überwinden und die prekäre Lage des Islam im bundesdeutschen Kirchenkartell $\ll^{47}$ durch eine striktere Trennung von Staat und Kirche zu beenden. Sie fordern eine neue Religionspolitik, d.h. Staat und Politik sollen durch politische Entscheidungen notfalls einseitig die bestehenden Regelungen verändern.

Solche politischen Entscheidungen im Rahmen des deutschen Parlamentarismus sind aber de facto Mehrheitsentscheidungen. Meine Politikfeldanalyse zu den sogenannten Kopftuch-Verboten verschiedener Bundesländer zeigt, dass diese »neue Religionspolitik « in der Realität keineswegs für eine politische Integration des Islam steht. ${ }^{48}$ Vielmehr hat die neue Religionspolitik teils gewollt, meist aber ungewollt laizistischen und ungewollt wohl auch islamistischen Kräften Aufwind verliehen. Vor allem aber hat sie auf

45 Vgl. Claus Leggewie, »Kirche oder Sekte? Muslime in Deutschland und den USA « in: Michael Minkenberg / Ulrich Willems (Hg.), Politik und Religion (= PVS- Sonderheft, Bd.33) Wiesbaden 2003, 164-183; Volker von Prittwitz, »Zivile oder herrschaftliche Religion? Fundamentalismus, Religionsfreiheit und Verantwortung des zivilen Staates « in: APuZ (2002) B18, 33-38; Ulrich Willems, »Weltanschaulich neutraler Staat, christlich-abendländische Kultur und Laizismus. Zu Struktur und Konsequenzen aktueller religionspolitischer Konflikte in der Bundesrepublik « in: Manfred Walther (Hg.), Religion und Politik. Zu Theorie und Praxis des theologisch-politischen Komplexes (= Schriftenreihe der Sektion Politische Theorien und Ideengeschichte in der DVPW, Bd.5) Baden-Baden 2004, 303-328; ders., "Reformbedarf und Reformfähigkeit der Religionspolitik in Deutschland « in: Zeitschrift für Politik 55 (2008), 64-83.

46 Willems, Staat, 304 u.ö.

47 Leggewie, Kirche, 174 (im Original als Überschrift hervorgehoben).

48 Vgl. Antonius Liedhegener, »Streit um das Kopftuch. Staat, Religion und Religionspolitik in der Bundesrepublik Deutschland « in: ZPol 15 (2005) H.4, 1181-1202; ders., »Religionsfreiheit und die neue Religionspolitik. Mehrheitsentscheide und ihre Grenzen in der bundesdeutschen Demokratie « in: Zeitschrift für Politik 55 (2008) 84-107. - Die in der Perspektive der Governance-Forschung geschriebene Studie von Julia von Blumenthal reflektiert bei aller Kenntnis im Detail kaum auf den religionspolitischen Kerngehalt des Problems der »Kopftuchverbote«, die bei ihr m.E. fälschlicherweise nur als auswechselbares Fallbeispiel von Gesetzgebung im bundesdeutschen Föderalismus vorgestellt werden. Vgl. Julia von Blumenthal, Das Kopftuch in der Landesgesetzgebung. Governance im Bundesstaat zwischen Unitarisierung und Föderalisierung (= Schriften zum Föderalismus, Bd.1), Baden-Baden 2009. 
Länderebene unterschiedliche Rechtsräume hervorgebracht, die de facto das Recht auf Religionsfreiheit vor allem für muslimische Frauen empfindlich einschränken.

Von diesen faktisch zu den Intentionen einer geforderten neuen Religionspolitik konträren Politikergebnissen einmal abgesehen, verkennt die These von der Notwendigkeit einer neuen staatlichen Religionspolitik die Bedeutung der friedensstiftenden Wirkung, die mit der wechselseitigen Verrechtlichung des Verhältnisses von Staat und Religionen auch in Deutschland eingetreten ist. In gewisser Weise endete das Zeitalter der so belastenden konfessionellen Spaltung in Deutschland erst mit dem Bonner Grundgesetz und der Etablierung der Bundesrepublik Deutschland als funktionierender Demokratie. Ganz ähnlich wie aus staatsrechtlicher Sicht sprechen somit auch politikwissenschaftliche Überlegungen dafür, das Erfolgsmodell der kooperativen Trennung behutsam fortzuschreiben. ${ }^{49}$

Dass sich diese Strategie im Blick auf den vielschichtigen, nicht kirchenförmig organisierten Islam schwieriger gestaltet, ist bekannt. Der Weg einer Einbeziehung in das kooperative Trennungsmodell kann aber zugleich als ein Weg zur sozialen und politischen Anerkennung der zahlreichen islamischen Verbände und Gruppen in die politische Gemeinschaft aufgefasst werden. In diese Richtung weist etwa die Deutsche Islamkonferenz. Aus Sicht des demokratischen Staates kann dies eine Einbindung in den demokratischen Grundkonsens und damit eine wünschenswerte Hegung bzw. Pazifizierung von »Religion « bedeuten. Die Alternative dazu ist die parteipolitische Politisierung von Religion im Ringen um Mehrheiten in Volksabstimmungen, Wahlen und Parlamenten. Europaweit hat dies zu einem anti-islamischen Populismus und zum Teil zur Bildung rechter Protestparteien geführt, was das gesellschaftliche und politische Klima einer freiheitlichen Demokratie vergiftet. Erinnert sei an das für den gegenwärtigen anti-islami-

49 Vgl. so schon Mathias Rohe, »Islam und deutsche Rechtsordnung. Möglichkeiten und Grenzen der Bildung islamischer Religionsgemeinschaften in Deutschland « in: Der Bürger im Staat 51 (2001), 233-240; ders., Der Islam - Alltagskonflikte und Lösungen. Rechtliche Perspektiven (= Herder Spektrum) Freiburg i. Br. u.a. 2001; Janbernd Oebbecke, »Tua Res Agitur. Die Rechte der Minderheitsreligionen und die Stellung der christlichen Kirchen. Warum die Diskussion über den Islam für die Kirchen wichtig ist « in: Wilhelm Geerlings / Thomas Sternberg (Hg.), Kirche in der Minderbeit. Sozialgeschichtliche Untersuchungen - pastorale Aspekte, Münster 2004, 105-135; ders., »Der Islam und die Zukunft des Verhältnisses zwischen Religionsgemeinschaften und Staat in Deutschland « in: Thorsten Schneiders / Lamya Kaddor (Hg.), Muslime im Rechtsstaat (= Veröffentlichungen des Centrums für Religiöse Studien Münster, Bd.3) Münster 2005, 131-144; ders., »Der Islam und die Reform des Religionsverfassungsrechts « in: Zeitschrift für Politik 55 (2008) 49-63. Allgemein zur Debatte Christian Walter, Religionsverfassungsrecht: in vergleichender und internationaler Perspektive (= Ius publicum, Bd.150) Tübingen 2006; Hans Michael Heinig / Christian Walter (Hg.), Staatskirchenrecht oder Religionsverfassungsrecht? Ein begriffspolitischer Grundsatzstreit, Tübingen 2006, und jüngst die Verhandlungen auf dem 68. Deutsche Juristentag in Berlin der Sektion Öffentliches Recht zum Thema »Öffentliches Recht. Neue Religionskonflikte und staatliche Neutralität - Erfordern weltanschauliche und religiöse Entwicklungen Antworten des Staates?«. 
schen Populismus vielfach stilbildende Plakat der Anti-Minarett-Kampagne 2009 in der Schweiz. ${ }^{50}$

Im Kern einer anzustrebenden gesellschaftlichen Einbeziehung, Hegung bzw. Pazifizierung von Religion steht die wechselseitige Anerkennung der Religionsfreiheit durch Staat und Religionsgemeinschaften. Vor allem Heiner Bielefeldt hat überzeugend argumentiert, dass das Grundrecht auf Religionsfreiheit in verfassungsstaatlichen Demokratien für Religionsgemeinschaften in einem spezifischen Sinne Bindung und Freiheit, $\mathrm{Zu}$ mutung und Chance zugleich bedeutet. ${ }^{51}$ Die Zumutung des säkularen Staates an jede Religionsgemeinschaft oder organisierte Weltanschauung besteht darin, dass der Staat im Interesse der Würde eines jeden Menschen davon absieht, religiöse oder weltanschauliche Wahrheitsfragen zur Entscheidung anzunehmen, zugleich aber einen unbedingten praktischen Geltungsvorrang des staatlichen Rechts einfordert und einfordern muss. ${ }^{52}$ Das Versprechen und die Leistung des säkularen Rechtsstaates ist im Gegenzug die Religionsund Bekenntnisfreiheit als Freiheit der freien Religionsausübung in individueller, kollektiver und korporativer Form.

\section{Religion und politische Entscheidungsprozesse: Macht und Einfluss kollektiver Akteure}

Macht ist eine der Grundkategorien der politischen Wissenschaft. So plausibel ihre Definition vor allem in der Tradition der Weber'schen Begrifflichkeit ist, so schwierig gestaltet sich weithin der empirische Nachweis von Macht und Einfluss in politischen Entscheidungsprozessen. Es überrascht daher nicht, in der bundesdeutschen Politikwissenschaft die Kategorie » Religion « traditionell vor allem in der Wahlforschung anzutreffen. Hier lassen sich anhand von Repräsentativumfragen religiöse Einflussfaktoren relativ exakt auf die Wahlentscheidung und damit auf die Machtverteilung in den Parlamenten beziehen. ${ }^{53}$ Religion und Wahlen sind und bleiben ein Dauerthema der politikwissen-

50 Das Plakat suchte beim Betrachter ein Bedrohungsszenario hervorzurufen, indem es eine in eine schwarze Burka gehüllte Frau vor einer Batterie raketenförmiger Minarette zeigte, welche eine Schweizer Fahne durchbohren.

51 Vgl. Heriner Bielefeldt, Muslime im säkularen Rechtsstaat. Integrationschancen durch Religionsfreibeit (= Global, local Islam), Bielefeld 2003.

52 »Dieser Vorrang besteht auch [...] gegenüber etwaigen Vorstellungen eines göttlichen Rechts.« Bielefeldt, Muslime, 33 und 124.

53 Mit Variationen gilt für bundesdeutsche Wahlen immer noch Franz Urban Pappis Diktum: »Wenn sich der Faktor Religion bei einer Bundestagswahl wieder als wichtig herausgestellt hat, tröstet sich mancher damit, daß dies demnächst ja anders werden müsse. Nur anders wird es nun schon seit 30 Jahren nicht. «Vgl. Franz Urban Pappi, »Konstanz und Wandel der Hauptspannungslinien in der Bundesrepublik « in: Joachim Matthes (Hg.), Sozialer Wandel in Westeuropa, Verhandlungen des 19. Deutschen Soziologentages 17.-20. April 1979 im Internationalen Congress Centrum (ICC) in Berlin, Frankfurt a.M/ New York 1979, 465-479, hier 472; Karl Schmitt, Konfession und Wablverhalten in der Bundesrepublik Deutschland (= Ordo Politicus, Bd.27), Berlin 1989; mit weiterer Literatur Harald Schoen, »Soziologische Ansätze in der empirischen Wahlforschung in: Jürgen W. Falter / Harald Schoen (Hg.), Handbuch Wablforschung, Wiesbaden 2005, 135-185. 
schaftlichen Forschung. ${ }^{54}$ Ähnlich, aber schon etwas komplizierter verhält es sich mit der Parteienforschung. Deren Interesse an Religion ist erst jüngst wieder erwacht. ${ }^{55} \mathrm{Ge}$ fragt wird, wie Parteien Religion benutzen, was religiöse Parteien ausmacht und ob die christdemokratischen Parteien zu den religiösen Parteien zu zählen sind. Letzteres hat Timotheos Frey in seiner vergleichenden Studie zur Christdemokratie in Westeuropa mit guten Gründen verneint. ${ }^{56}$

Weitaus schwieriger gestaltet sich die Erforschung des politischen Einflusses von Religionsgemeinschaften und ihren Organisationen auf parlamentarisch-politische Entscheidungen. Hier teilt die politische Soziologie von Religion und Kirchen die Schwierigkeiten der Verbände- und Interessenforschung. ${ }^{57}$ Fest steht, dass die von Casanova so vehement geforderte Abstinenz religiöser Akteure in der Machtpolitik mit den Realitäten etwa in den USA oder der Bundesrepublik Deutschland nicht zur Deckung zu bringen ist. ${ }^{58}$ Zahlreich sind die Studien zur Politikfähigkeit von Religion in politischen Systemen. Das Zwischenfazit der bisherigen Forschung lautet: Religiöse Akteure beteiligen sich in Demokratien an Politik oftmals in einem ganz handfestem Sinn. Sie formulieren Interessen und machen Politik für sich, stellvertretend für andere, meist Schwächere der Gesellschaft, sowie für das Gemeinwohl der politischen Gemeinschaft insgesamt.

Die theoretisch zentrale Frage lautet, ob es ein Modell gibt, das wenn auch nicht als kausale Erklärung, so doch als Heuristik in der Lage ist, die Voraussetzung und Erfolgsbindungen für religiöse Akteure in einem gegebenen politischen System zu erklären. Dazu gibt es zumindest einige Vorschläge. Ausgehend von dem bekannten strukturellfunktionalen Politikmodell in der Tradition von David Easton und Gabriel Almond kann man einen beliebigen kollektiven Akteur zunächst selbst als ein politisches Subsystem

54 Heute bedarf dies mehr denn je auch der transnationalen Perspektive. Vgl. David Broughton / Hans Martien ten Napel (Hg.), Religion and Mass Electoral Behaviour in Europe (= Routledge/ ECPR Studies in European Political Science, Bd.19) London - New York 2000; Karsten Grabow / Pattrick Köllner (Hg.), Parteien und ibre Wähler. Gesellschaftliche Konfliktlinien und Wählermobilisierung im internationalen Vergleich, St. Augustin / Berlin 2008.

55 Vgl. als Überblick Payam Mohseni / Clyde Wilcox, »Religion and Political Parties « in: Jeffrey Haynes (Hg.), Routledge Handbook of Religion and Politics (= Routledge International Handbooks), New York, NY 2009, 211-230; Stathis N. Kalyvas / Kees van Kersbergen, »Christian Democracy« in: Annual Review of Political Science (2010) 183-209.

56 Vgl. Timotheos Frey, Die Christdemokratie in Westeuropa. Der schmale Grat zum Erfolg, Baden-Baden 2009, bes. 47-56. Vgl. auch Udo Zolleis, Die CDU. Das politische Leitbild im Wandel der Zeit, Wiesbaden 2008, 13-24 und 89-96.

57 Vgl. mit weiterer Literatur zum Problemkontext Antonius Liedhegener, Macht, Moral und Mebrbeiten. Der politische Katholizismus in der Bundesrepublik Deutschland und den USA seit 1960 (= Jenaer Beiträge zur Politikwissenschaft, Bd.11) Baden-Baden 2006, 24-19; Ulrich Willems, »Kirchen« in: Thomas von Winter / Ulrich Willems (Hg.), Interessenverbände in Deutschland, Wiesbaden 2007, 316-340.

58 Vgl. explizit zu Casanovas Ansatz Antonius Liedhegener, » Plural und politisch. Der Katholizismus in der Bundesrepublik Deutschland seit 1989/90 « in: Jabrbuch für Christliche Sozialwissenschaften 44 (2003), 53-72; Karl Gabriel / Hans-Joachim Höhn (Hg.), Religion beute öffentlich und politisch. Provokationen, Kontroversen, Perspektiven, Paderborn 2007; Corwin Smidt / Lyman A. Kellstedt / James L. Guth (Hg.), The Oxford Handbook of Religion and American Politics, Oxford - New York 2009. 
auffassen, wenn er Politik mitzugestalten sucht. Das bedeutet, dass Prozesse erfolgreicher interner politischer Willensbildung die wesentliche Voraussetzung dafür sind, damit ein solcher kollektiver Akteur überhaupt mit Aussicht auf Erfolg an jenem übergeordneten politischen Entscheidungsprozess teilnehmen kann, der auf die Herstellung gesamtgesellschaftlich verbindlicher Entscheidungen zielt.

Gibt es aber Spezifika religiöser Akteure, die in die Modellbildung für den hier interessierenden Fall Religion unbedingt mit einfließen müssen? Diese Frage hat Robert Fowler in seinem Konzept ausdrücklich bejaht. ${ }^{59}$ Politischer Einfluss von Religionen entsteht nach ihm in einem mehrstufigen Prozess. An dessen Basis steht ein religiöses bzw. theologisches Programm des jeweiligen Akteurs. Glaubensaussagen und Theologien sind danach also konstitutiv. Über den politischen Erfolg insgesamt entscheiden aber auch und vor allem externe Faktoren, d.h. Rahmenbedingungen, die der jeweilige Akteur nur bedingt zu gestalten vermag. Darüber hinaus macht Fowler deutlich, dass sich die politischen Positionen, Interessen und Forderungen einer Religion nicht allein und auch nicht vorrangig durch den Rückgriff auf heilige Texte oder dogmatische Lehraussagen ermitteln lassen. Im Zentrum des politikwissenschaftlichen Interesses muss vielmehr die je zeitgebundene 'Theologie des Politischen', d.h. die konkrete Anwendung religiöser Überzeugungen und Vorstellungen in einer gegebenen politischen Konstellation stehen. Ob etwa der Islam demokratiefähig ist oder nicht, lässt sich also nicht am Koran und der Scharia allein entscheiden. Und die Geschichte der Katholischen Kirche zwischen der Französischen Revolution und dem Zweiten Vatikanischen Konzil unterstreicht die Wandlungsfähigkeit religiöser Traditionen auch und gerade in politischer Hinsicht. ${ }^{60}$

Entwickelt anhand der Erfahrungen mit dem präsidentiellen Regierungssystem der USA stellt Fowlers Modell insbesondere die Funktionsunterschiede zwischen verschiedenen Typen von Demokratien zu wenig in Rechnung. Für ländervergleichende Studien kommt es aber darauf an, sowohl die externen Bedingungen für politischen Erfolg systematisch zu berücksichtigen, die das Regierungssystem durch seine strukturellen Handlungsimperative und die jeweils herrschenden (partei-)politischen Machtverhältnisse setzt, als auch die langfristigen (zeit-)historischen Rahmenbedingungen sowie die internen Handlungsbedingungen von Religionsgemeinschaften in die Analyse explizit einzubeziehen. Fowlers fruchtbares Modell sollte daher um die genannten Faktorenbündel erweitert

59 Vgl. Robert Booth Fowler, Religion and Politics in America, Metuchen, NY 1985, 167-175; ders. (u.a.), Religion and Politics in America. Faith, Culture, and Strategic Choices, 3. Aufl., Boulder, CO - Oxford 2004, 61-64.

60 Vgl. dazu zuletzt Karl Gabriel / Christian Spieß / Katja Winkler (Hg.), Religionsfreibeit und religiöser Pluralismus. Entwicklungslinien eines katholischen Lernprozesses (= Katholizismus zwischen Religionsfreiheit und Gewalt, Bd.1) Paderborn u.a. 2010. 


\section{Abb. 1: Modell mit vier Faktorenbündeln zur Erklärung politischen Erfolgs von religiösen Gruppierungen in Demokratien}

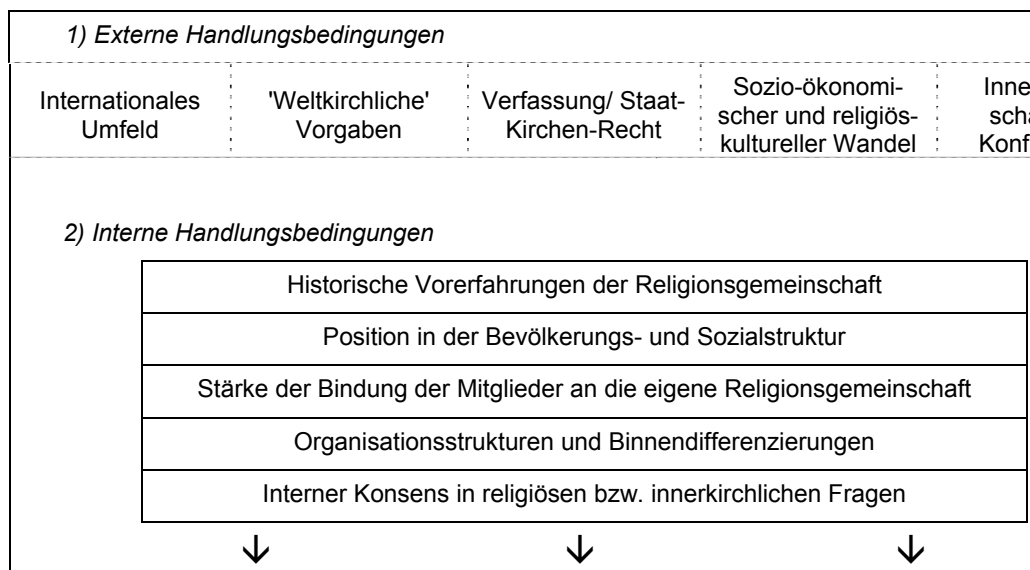

3) Interne Erfolgsbedingungen

\begin{tabular}{|c|}
\hline Politische \\
Einstellungen \\
der Mitglieder \\
\hline
\end{tabular}

$y$

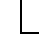

Theologie des
Politischen der
Religionsgem.

$\downarrow$
Innergesellschaftliche Konfliktlinien
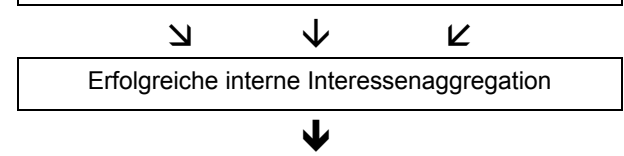

Ressourcen politischer Interessenvertretung

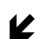

4) Externe Erfolgsbedingungen

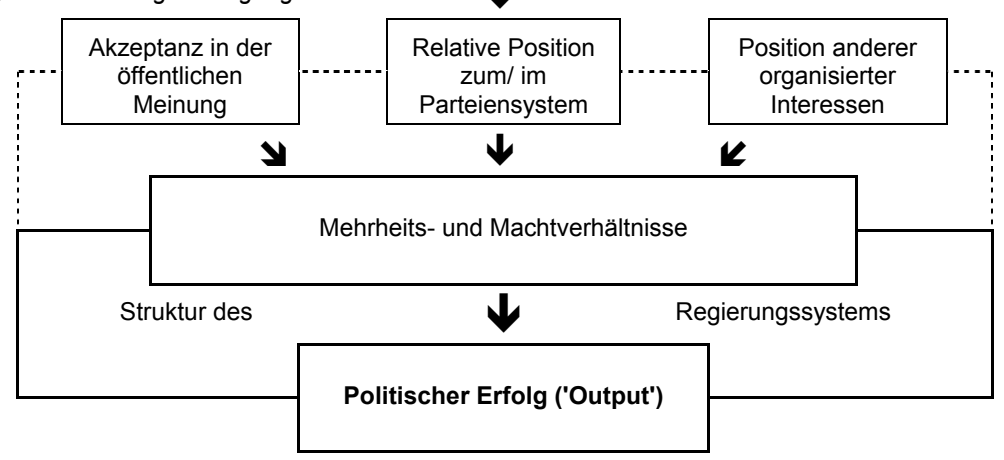

Quelle: Inhalt und Darstellung A.L. 
werden (Abb. 1). ${ }^{61}$ Ohne einen solchen theoretisch-konzeptionellen Rahmen wird es nämlich nicht gelingen, den Zusammenhang von Religion und politischem Einfluss in politischen Systemen vergleichend $\mathrm{zu}$ erforschen und $\mathrm{zu}$ erfassen. Dies wird insbesondere dann wichtig, wenn es gilt, die Rolle von Religion und religiösen Akteuren in zentralen Politikfeldern wie der Lebensschutzpolitik, der Sozialpolitik, der Familienpolitik, der Gentechnik, der Europapolitik oder der Außen- und Sicherheitspolitik für die Gegenwart empirisch zu bestimmen. ${ }^{62}$

\section{Religion und Zivilgesellschaft: die Integration pluraler Gesellschaften}

»Der Katholizismus dringt in Norddeutschland vor: Kirchenbauten wohin man sieht, auch in Gegenden mit kaum nennenswerter katholischer Bevölkerung «. ${ }^{63}$ - Shortly after the war, labor unions [the Knights of the Southern Cross] were formed to protect the interests of Catholics [...]: those were the days when job advertisments frequently carried the advice >Catholics need not apply< « ${ }^{64}$ Diese beiden für uns heute befremdlichen Zitate - das erste von 1963 aus Niedersachen, das zweite aus Australien nach 1918 - weisen darauf hin, dass die Erfahrung religiös konnotierter Überfremdungsängste und die Erfahrung sozialer Diskriminierung auf der Grundlage religiöser Zugehörigkeiten zeit- und kulturübergreifende Phänomene sind. Es wäre nicht schwer, ähnliche Aussagen für den Bau von Moscheen oder die beruflichen Chancen islamischer Jugendlicher in Deutschland heute beizubringen. Religionszugehörigkeit kann in hohem Maße über die gesellschaftliche Position Einzelner wie sozialer Gruppen mitentscheiden. Die gut erforschte Geschichte der konfessionellen und sozialen Großmilieus in der deutschen Geschichte

61 Vgl. zu diesem erweiterten Modellvorschlag, der das Ergebnis induktiver Theoriebildung an den Fallbeispielen des Katholizismus in der Bundesrepublik Deutschland und den USA ist, Liedhegener, Macht, Moral und Mebrbeiten (FN57), 29-39 und 442-446 und ders., »Macht und Einfluss von Religionen. Theoretische Grundlagen und empirische Befunde der politischen Systemlehre und politischen Kulturforschung « in: Antonius Liedhegener / Andreas TungerZanetti / Stephan Wirz (Hg.), Religion - Wirtschaft-- Politik. Forschungszugänge zu einem aktuellen transdisziplinären Feld (= Religion - Wirtschaft - Politik) Baden-Baden - Zürich 2011, 241-273.

62 Das Interesse an solcherart Zusammenhängen ist in den letzten Jahren erkennbar gewachsen. Vor allem die vergleichende Wohlfahrtsstaatforschung hat hierzu einen wichtigen Beitrag geliefert. Vgl. Francis G. Castles (Hg.), Families of Nations, Patterns of Public Policy in Western Democracies, Aldershot 1993; ders., "On Religion and Public Policy: Does Catholicism Make a Difference? « in: European Journal of Political Research 25 (1994) 19-40; Philip Manow, Religion und Sozialstaat. Die konfessionellen Grundlagen europäischer Woblfahrtsstaatsregime (= Theorie und Gesellschaft, Bd.68), Frankfurt a. M. 2009; Kees van Kersbergen / Philip Manow (Hg.), Religion, Class Coalition and the Welfare States (= Cambridge Studies in Social Theory, Religion, and Politics), Cambridge 2009.

63 Qualitative EMNID-Untersuchung »Motive und Einstellungen der niedersächsischen Bevölkerung. Eine Untersuchung zur bevorstehenden Landtagswahl 1963«, 25, zit. nach Frank Bösch, Die Adenauer-CDU. Gründung, Aufstieg und Krise einer Erfolgspartei 1945-1969, Stuttgart - München 2001, 339.

64 Robert E. Dixon, The Catholic Community in Australia (= Australia's Religious Communities) Adelaide 2005, 5. 
zeigt dies exemplarisch. ${ }^{65}$ Umstritten ist nach wie vor, ob die Ausbildung religiöser oder sozialer Großmilieus - die Öffentlichkeit spricht derzeit gerne von Parallelgesellschaften - im Blick auf die Integration und Stabilität von Gesellschaften und politischen Systemen Fluch oder Segen ist. Es handelt sich offenkundig um ein zutiefst ambivalentes Phänomen.

Eine trennschärfere Klärung kann eine etwas andere theoretische Einordung von Religionsgemeinschaften und ihren zahlreichen Organisationen liefern. Die jüngere sozialwissenschaftliche Forschung plädiert vielfach dafür, Religion als möglichen Teil der Bürger- bzw. Zivilgesellschaft aufzufassen. Für die USA ist diese Zuordnung eine unumstrittene, tief in der demokratischen Tradition des Landes verankerte Tatsache. Hier sind Vereine, Freiwilligenarbeit und bürgerschaftliches Engagement beinahe von Anfang an zentraler Bestandteil der Selbstorganisation und des Selbstverständnisses der amerikanischen Gesellschaft. Man ist stolz, eine »nation of joiners « zu sein. Religion und religiöse Organisationen sind integraler und wesentlicher Bestandteil der Zivilgesellschaft. Schon Tocqueville hat dies beschrieben. In Deutschland mit seinen staatskirchlichen Traditionen vor allem im Protestantismus stößt die Zuordnung von Kirchen und Religionsgemeinschaften zur Zivilgesellschaft verschiedentlich noch auf Vorbehalte. Jüngst hat daher Paul Nolte, der sich bewusst in die Tradition evangelischen Glaubens in Deutschland stellt, nachdrücklich dafür plädiert, Religion und Kirchen auch hier als zivilgesellschaftliche Ressource zu betrachten. ${ }^{66}$

In den USA reagiert die Öffentlichkeit vor dem Hintergrund des hohen Stellenwerts von Freiwilligenarbeit und bürgerschaftlichem Engagement auf Alarmrufe eines angeblichen Verfalls der Zivilgesellschaft schnell und besonders besorgt. Der Aufsatz »Bowling alone « war 1995 ein solcher Alarmruf. ${ }^{67}$ Robert Putnam führte darin eindrückliche Zahlenreihen zum Niedergang des bürgerschaftlichen Engagements in den USA seit den 1960er Jahren vor. Sein Beitrag löste eine breite, bald über die Grenzen der eigenen Nation hinausreichende Debatte aus. Zwar galten die Sorgen Putnams für die USA rasch als überzogen. ${ }^{68}$ Bleibende Bedeutung gewann Putnam aber nicht zuletzt dadurch, den Begriff des »Sozialkapitals« und die zentrale Rolle der Religion für die Bildung von Sozi-

65 Vgl. bilanzierend Christoph Kösters / Antonius Liedhegener, »Historische Milieus als Forschungsaufgabe. Zwischenbilanz und Perspektiven « in: Westfälische Forschungen 48 (1998) 593-601; Peter Lösche / Franz Walter, »Katholiken, Konservative und Liberale: Milieus und Lebenswelten bürgerlicher Parteien in Deutschland während des 20. Jahrhunderts « in: Geschichte und Gesellschaft 26(2000) 471-492; Christoph Kösters / Antonius Liedhegener / Wolfgang Tischner / Claudio Kullmann, »Was kommt nach dem katholischen Milieu? Forschungsbericht zur Geschichte des Katholizismus in Deutschland in der zweiten Hälfte des 20. Jahrhunderts« in: AfS 49(2009) 485-526.

66 Vgl. Paul Nolte, Religion und Bürgergesellschaft. Brauchen wir einen religionsfreundlichen Staat? (= Berliner Reden zur Religionspolitik) Berlin 2009.

67 Robert D. Putnam, »Bowling Alone: America's Declining Social Capital in: Journal of Democracy 6 (1995), 64-78; ders., Bowling Alone. The Collapse and Revival of American Community, New York u. a. 2000.

$68 \mathrm{Vgl}$. Alan Wolfe, »Is Civil Society Obsolete? Revisiting Predictions of the Decline of Civil Society in Whose Keeper? « in: E. J. Dionne Jr., (Hg.), Community Works. The Revival of Civil Society in America, Washington, DC 1998, 17-23, hier 21. 
alkapital in der Forschung etabliert zu haben. ${ }^{69} \mathrm{Zu}$ Recht ist in der deutschen Forschung darauf hingewiesen worden, dass die Faszination des Begriffs darauf beruht, dass das Sozialkapital Putnam'scher Prägung konzeptionell eine Brücke vom autonomen Individuum zum potentiell gemeinwohlorientierten kollektiven Denken und Handeln schlägt. ${ }^{70}$ In der aktuellen amerikanischen Forschung zum Sozialkapital haben sich gerade die Kirchengemeinden als ein zentraler Ort zivilgesellschaftlichen Engagements erwiesen. Die Literatur dazu ist mittlerweile zahlreich. ${ }^{71}$ Nun könnte man geneigt sein, diese Tatsache der großen Bedeutung von Religion für die Zivilgesellschaft in den USA einmal mehr als singulär, als Ausdruck des bekannten American Exceptionalism auch in religiöser Hinsicht deuten.

Eine solche Deutung ginge aber fehl. Zu äußerst interessanten Ergebnissen sind nämlich Pippa Norris und Ronald Inglehart gekommen. Sie haben anhand der Daten des World Value Surveys auch nach dem Zusammenhang von Religion und bürgerschaftlichem Engagement gefragt. Berücksichtigt wurden rund 80 Länder. Ausgewertet wurden die Antworten von zehntausenden von Befragten aus allen Kulturkreisen dieser Erde auf die Frage, ob sie sich in einem Verein oder Ähnlichem engagieren. Ihre Antworten wurden dann differenziert nach der Häufigkeit der Teilnahme an Gottesdiensten. In dieser Auswertung zeigen sich klare, signifikante Differenzen in zahlreichen Kategorien zivilgesellschaftlichen Engagements wie Frauengruppen, Jugendarbeit, local community action, Sozialarbeit und Kultur. Je höher der Gottesdienstbesuch, desto höher das zivilgesellschaftliche Engagement. »The pattern confirms social capital theory's claim that the social networks and personal communications derived from regular churchgoing play an important role.$^{72}$

69 Nach einer Definition Putnams bedeutet Sozialkapital »connections among individuals - social networks and the norms of reciprocity and trustworthiness that arises from them. « Putnam, Collapse (FN 67), 19.

$70 \mathrm{Vgl}$. Annette Zimmer, Vereine - Zivilgesellschaft konkret, unter Mitarbeit von Thorsten Hallmann und Lilian Schwab (= Grundwissen Politik, Bd.16), 2. Aufl., Wiesbaden 2007, 198-199; Bettina Westle, Oscar W. Gabriel, (Hg.), Sozialkapital. Eine Einführung (= Studienkurs Politikwissenschaft), Baden-Baden 2008, 11-50.

71 Vgl. mit zahlreichen weiteren Nachweisen etwa Corwin Smidt (Hg.), Religion as Social Capital. Producing the Common Good, Waco, TX 2003; Mark Chaves, Congregations in America, Cambridge - London 2004;Antonius Liedhegener / Werner Kremp (Hg.), Civil Society, Civic Engagement and Catholicism in the U.S. (= Atlantische Texte, Bd.27) Trier 2007; Robert Wuthnow, »Religious Involvement and Status-Bridging Social Capital in: Journal for the Scientific Study of Religion 41 (2002), 669-684; Nancy Tatom Ammerman, Pillars of Faith. American Congregations and Their Partners, Berkeley/Los Angeles/London 2005; Antonius Liedhegener, Art. »Churches and Denominations «, in: Helmut K. Anheier / Stefan Toepler / Regina List, (Hg.), International Encyclopedia of Civil Society, New York 2010, 133-138.

72 Norris/ Inglehart, Religion, 191.

ZfP 58. Jg. 2/2011 
Abb. 2: Kirchenbindung und zivilgesellschaftliches Engagement (Zusammensetzung der Bevölkerung bzw. Engagementgruppen nach Kirchenbindung in Prozent)
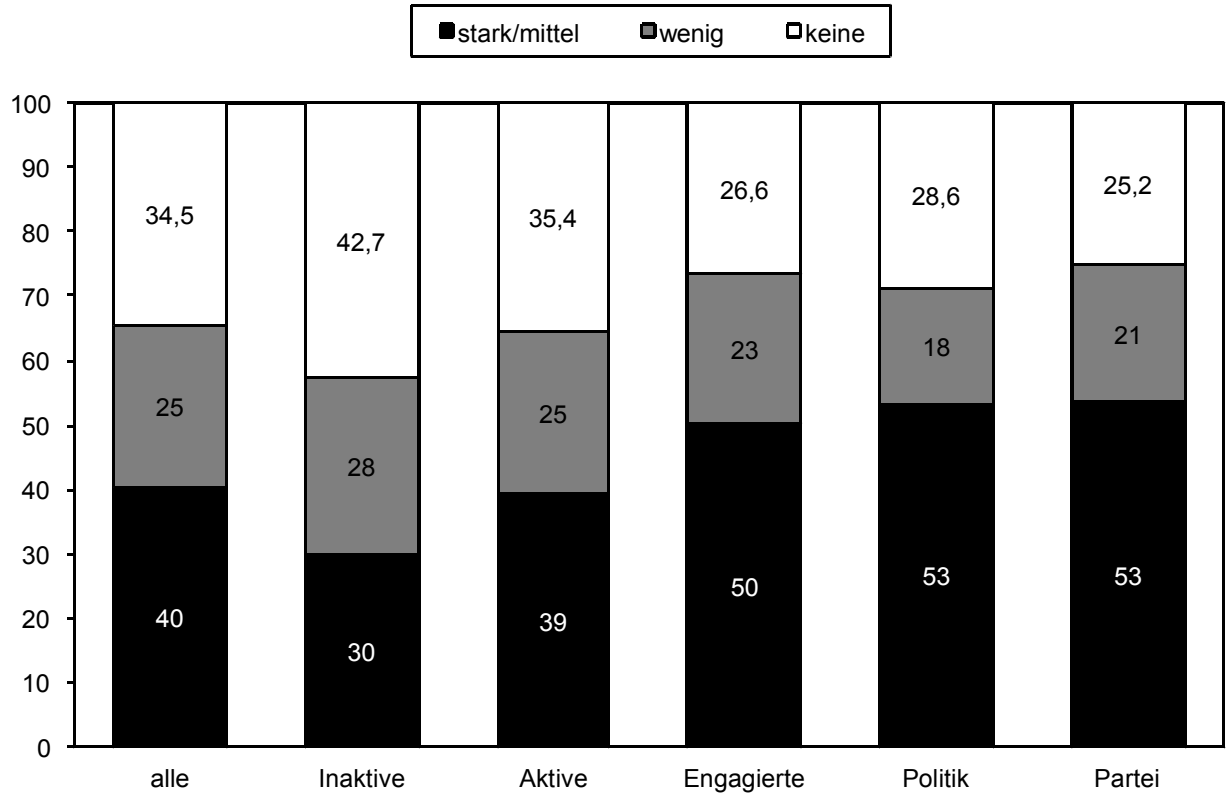

Bemerkung: Alle $=$ Zusammensetzung der Bevölkerung; Inaktive $=$ keinerlei aktive Einbindung in zivilgesellschaftliche Organisationen bzw. Initiativen; Aktive = aktives Mittun in Vereinen etc.; Engagierte $=$ bürgerschaftlich, aber nicht politisch Engagierte; Politik = politisch außerhalb von Parteien Engagierte; Partei = parteipolitisch Engagierte. - Lesehilfe: Befragte mit einer starken oder mittleren Kirchenbindung stellen 53 \% aller parteipolitisch Engagierten.

Quelle: Eigene Berechnungen A.L. nach Primärdatensatz Freiwilligensurvey 2004 (ZA4331)

Lässt sich ein solcher Zusammenhang generell auch in anderen hochmodernen Gesellschaften ausmachen? In dieser Frage gehen die Befunde der internationalen empirischen Forschung derzeit auseinander. ${ }^{73}$ Bei den aktuellen Forschungsergebnissen zur Bundesrepublik Deutschland zeigt sich allerdings eine Konvergenz der Befunde. Die vorliegenden Daten zeigen im Grundzug exakt den gleichen Zusammenhang wie die weltweiten Daten. Mit steigender Bindung an eine Religionsgemeinschaft nimmt die Häufigkeit der

73 Vgl. Antonius Liedhegener / Ines-Jacqueline Werkner, »Religion, Zivilgesellschaft und politisches System - ein offenes Forschungsfeld « in: Antonius Liedhegener / Ines-Jacqueline Werkner (Hg.), Religion zwischen Zivilgesellschaft und politischem System. Befunde-Positionen-Perspektiven (= Politik und Religion) Wiesbaden 2011, 9-36, hier 19-24. 
aktiven Mitgliedschaft in zivilgesellschaftlichen Organisationen deutlich zu. ${ }^{74}$ Religionszugehörigkeit bzw. Religiosität und die Dichte der Netzwerke bürgerschaftlichen Engagements hängen also auch in Deutschland eng zusammen. Das gilt auch und gerade für das politische und parteipolitische Engagement in Deutschland (Abb. 2).

\section{Fazit: Befunde und Politikempfehlungen einer verstehenden politischen Soziologie von Religion und Kirchen}

Angesichts des drastischen Bedeutungsgewinns des Zusammenhangs von Politik und Religion in der Gegenwart und einer zunehmend unübersichtlichen Forschungslage war es das Ziel des vorliegenden Beitrags, einen Problemaufriss anzubieten, der die Schwerpunkte der derzeitigen Forschung sichtbar macht und diese konzeptionell bündelt. Dazu wurden hier vier Problemkreise definiert und einschlägige Forschungen dazu vorgestellt.

Der erste Problemkreis steht für den von der Öffentlichkeit besonders stark wahrgenommenen Zusammenhang von Religion und politischer Gewalt. Die bisherigen Forschungen zum »Fundamentalismus", die am Anfang des neuen Interesses am Wechselverhältnis von Politik und Religion gestanden haben, konvergieren darin, dass es sich bei fundamentalistischen Kreisen und Bewegungen um eine Aneignung religiöser Traditionen mit dem Ziel handelt, einer als verdorben wahrgenommenen Moderne eine reine Lehre entgegenzustellen, die Alltag und Politik der Anhänger und ggfs. der Herrschaftsunterworfenen bestimmen soll. Die religiöse Militanz von Fundamentalismen bedeutet nicht immer und nicht automatisch eine Bereitschaft, physische politische Gewalt und terroristische Mittel einzusetzen; ein innerer Zusammenhang ist gleichwohl unverkennbar. Rechtsstaatliche Demokratien sollten und müssen hier ihr Gewaltmonopol in die Waagschale werfen und innen- wie außenpolitisch eine klare Grenze zur physischen Gewalt und verbalen Radikalisierung ziehen. Hier kann es keine kulturalistische Anerkennung geben. In den gleichen Problemkreis gehört die Einsicht, dass Religionen in kriegerischen Konflikten der Gegenwart eine eigenständige Rolle spielen können, in vielen Fällen ihnen aber weniger die Rolle einer Erstursache als die einer Verstärkerfunktion etwa in ethnischen Konflikten zukommt. Handlungspraktisch empfehlen jene, die diesen

74 So übereinstimmend Thomas Gensicke / Sibylle Picot / Sabine Geiss, Freiwilliges Engagement in Deutschland 1999-2004. Ergebnisse der repräsentativen Trenderbebung zu Ehrenamt, Freiwilligenarbeit und bürgerschaftlichem Engagement, in Auftrag gegeben und hg. vom Bundesministerium für Familie, Senioren, Frauen und Jugend, vorgelegt von TNS Infratest Sozialforschung, Wiesbaden 2006, 88-91; Volkhard Krech, »Exklusivität, Bricolage und Dialogbereitschaft. Wie die Deutschen mit religiöser Vielfalt umgehen « in: BertelsmannStiftung (Hg.), Religionsmonitor 2008, Gütersloh 2007, 33-43, hier 42; Antonius Liedhegener, » Linkage im Wandel. Parteien, Religion und Zivilgesellschaft in der Bundesrepublik Deutschland « in: Antonius Liedhegener / Ines- Jacqueline Werkner (Hg.), Religion zwischen Zivilgesellschaft und politischem System. Befunde - Positionen - Perspektiven (= Politik und Religion) Wiesbaden 2011, 232-257; Sigrid Roßteutscher, Religion, Zivilgesellschaft, Demokratie. Eine international vergleichende Studie zur Natur religiöser Märkte und der demokratischen Rolle religiöser Zivilgesellschaften (= Studien zur Wahl- und Einstellungsforschung, Bd.12), Baden-Baden 2009; Richard Traunmüller, »Religion und Sozialintegration. Eine empirische Analyse der religiösen Grundlagen sozialen Kapitals« in: Berliner Journal für Soziologie 19 (2009) H. 3, 435-468. 
Zusammenhang untersucht haben, daher vor allem, die friedensförderlichen Potentiale von Religion in der religiösen wie staatlichen Unterweisung zu stärken und zu fördern, um Religionen und ihre Anhänger »gewaltresistent« zu machen.

Der zweite Problemkreis »Religion und politische Herrschaft« fokussiert die politisch-rechtlichen Beziehungen zwischen staatlicher Herrschaft und Religionsgemeinschaften. In der zweiten Hälfte des 20. Jahrhunderts sind viele mögliche Konfliktpunkte zwischen Staat und Kirchen bzw. Religionsgemeinschaft zunächst verrechtlicht worden, was durchaus zu einer deutlichen Befriedung älterer religionspolitischer Konflikte beigetragen hat. Eine Trennung von Staat und Kirche in freundlicher oder kooperativer Form war Voraussetzung und Resultat zugleich. Dieses klassische Staat-Kirche-Verhältnis ist heute erkennbar in Bewegung geraten. Sichtbarster Ausdruck sind Forderungen von Politikwissenschaftlern nach einer neuen Religionspolitik, die sich in Deutschland im Namen der Integration des Islam gegen die bestehenden Strukturen wenden. Die tatsächlichen Ergebnisse der neuen Religionspolitik durch Parlamentsbeschlüsse und Volksentscheide sind - wie gesehen - allerdings ernüchternd: Kopftuch- und Minarettverbote beschränken und verringern als solche nicht nur die Freiheitsräume individueller Religionsausübung, sondern tendieren überdies dazu, eine neue parteipolitische Konfliktlinie zu schaffen, deren Beherrschbarkeit im Rahmen politisch verhandelbarer, die verfassungsmäßige Ordnung nicht destabilisierender Konflikte bezweifelt werden muss. Religionspolitik per Mehrheitsentscheidung wirkt sich also in mehrfacher Hinsicht problemverschärfend aus.

Im dritten Problemkreis »Religion und politische Entscheidungsprozesse « wurde anhand der vorliegenden jüngeren Studien sichtbar, dass religiöse Organisationen als 'public religions' keineswegs auf den Raum der zivilgesellschaftlichen Öffentlichkeit bzw. Debatte beschränkt sind. In organisierter Form sind und waren Kirchen, kirchliche Vereine, Verbände und Einrichtungen und andere große und kleinere religiöse Gemeinschaften stets Teil demokratischer Willensbildungs- und Entscheidungsprozesse. Ob, wie und wofür sie sich politisch engagieren, ist nicht unwesentlich von ihrem theologischen Profil und Selbstverständnis abhängig. Handlungsleitend sind aber nicht heilige Texte oder abstrakte Glaubensnormen, sondern die in konkreten Zeitumständen vertretene Aktualisierung und Kontextualisierung von Glaubensvorstellungen, die hier als Theologie des Politischen bezeichnet wurde. Darüber hinaus teilt die politische Soziologie von Religion und Kirchen hier die Schwierigkeiten der Verbände- und Interessenforschung: Politische Macht und politischer Einfluss sind theoretisch nur schwer zu modellieren und empirisch schwierig zu erforschen. Modellangebot und empirische Studien dazu gibt es aber. Danach ist eine solche politische Rolle von religiösen Akteuren im Rahmen allgemeiner demokratischer Willensbildungs- und Entscheidungsprozesse in der Regel ebenso unspektakulär wie für die Gemeinwohlfindung förderlich und eventuell sogar unverzichtbar. Politisch-praktisch bedarf es dazu eines strukturierten Dialogs, wie die jüngsten Entwicklungen in der EU belegen.

Und schließlich ist Religion zentral für das Thema der Integration moderner Gesellschaften. Dabei ist zunächst weniger an die so oft geforderte Integration Einzelner oder spezieller Gruppen in eine gegebene Gesellschaft gedacht; hier kann und wird es in plu- 
ralen Gesellschaften im Rahmen der demokratischen Grundordnung Spannungen zwischen verschiedenen Vorstellungen und Lebensweisen geben. Vielmehr geht es um die Frage der Integration auf einer systemischen, gesamtgesellschaftlichen Ebene. Stärker als noch vor 15 oder 20 Jahren wird dafür heute neben dem staatlich-politischen Handeln die Bedeutung der Zivilgesellschaft gesehen. Empirisch scheint derzeit in vielen Gesellschaften zu gelten: Religionen und deren Zusammenschlüsse stärken in der Summe die Zivilgesellschaft. Dieser Zusammenhang ist aber kein theoretisch zwingender, sondern ein kontingenter. Wenn Religionsgemeinschaften und ihre Aktivitäten faktisch einen Beitrag zur Integration moderner Gesellschaften liefern, ist es Aufgabe von Forschung und Politik darüber nachzudenken, unter welchen Bedingungen dies geschieht. Ein anhaltender Kirchenschwund könnte dann durchaus auch gesellschaftlich bedrohliche Züge haben, wäre also nicht nur eine Angelegenheit der Kirchen und Glaubensgemeinschaften selbst.

In der Zusammenschau der Befunde zu den vier grundlegenden Problemkreisen des Verhältnisses von Politik und Religion wird erkennbar, dass die praktische Politik im Blick auf die neue religiöse Vielfalt gegenwärtig in ein sehr schwierig zu gestaltendes Feld gestellt ist. Das Gewaltpotential von Religionen im Allgemeinen wie gegenwärtig von Teilen des Islam im Besonderen erfordert in verfassungsstaatlichen Demokratien ohne Zweifel staatliche Wachsamkeit und Gefahrenabwehr. Gleichzeitig muss das politische System in der Lage sein, auf die berechtigten Interessen religiöser Minderheiten auch gegen den öffentlichen Druck durch mediale Berichterstattung, säkularistische Gruppierungen und populistische Mehrheiten einzugehen und diese Interessen gegebenenfalls zu schützen und zu fördern. Das erfordert einen behutsamen religionspolitischen Umgang mit Verfassung und Recht, eine wechselseitige Bereitschaft, auf der Basis der Religionsfreiheit und der grundsätzlichen Trennung von Staat und Religionen Formen der institutionellen Kooperation zu schaffen, sowie generell die zivilgesellschaftlichen Potentiale von Kirchen und Religionsgemeinschaften politisch ernst zu nehmen und zumindest nicht zu untergraben. Politikwissenschaftliche Forschung kann dabei helfen, religionspolitische Handlungsbedingungen und deren Entscheidungskorridore zu erhellen. Darüber hinaus sind aber der politische Wille der Bürgerinnen und Bürger, für eine freiheitliche Demokratie einzustehen, und die Klugheit und Kunst der Politikerinnen und Politiker, gerade in der gegenwärtig komplexen religionspolitischen Lage umsichtig zu entscheiden, gefordert. Das Verhältnis von Politik und Religion ist und bleibt für Politik und politische Wissenschaft eine wichtige Querschnittsaufgabe.

\section{Zusammenfassung}

Weltweit nimmt der Einfluss der Religionen auf Politik und Gesellschaft zu. Selbst im vermeintlich säkularen Europa hat das Spannungsfeld von Politik und Religion erheblich an Bedeutung gewonnen. Im Zentrum des öffentlichen Interesses steht dabei meist das Konflikt- und Gewaltpotential von Religion. Das Wechselverhältnis von Politik und Religion ist aber weitaus vielschichtiger, als es das vorherrschende Medienbild der reli- 
giös motivierten Gewalt und des islamistischen Terrors vermittelt. In den aktuellen Befunden der politischen Wissenschaft, die sich nach längerer Zeit der Abstinenz dem Verhältnis von Politik und Religion wieder vermehrt widmet, spiegelt sich diese Vielschichtigkeit. Zugleich ist hier aber auch eine gewisse Unsicherheit zu erkennen, wie mit dem Phänomen Religion analytisch umzugehen ist. Der Beitrag unternimmt den Versuch, die neu entstandene, unübersichtliche Forschungslandschaft zu systematisieren und so Religion in ihren vielfältigen Bezügen zur Politik in einen stimmigen Analyserahmen der politischen Wissenschaft zu bringen. Insgesamt erweist sich das Verhältnis von Politik und Religion als eines der großen Querschnittsthemen einer politischen Wissenschaft, deren Befunde die Handlungsmöglichkeiten demokratischer Politik erhellen können.

\section{Summary}

The influence of religions on politics and society is growing globally. Even in the presumed secular Europe, the area of conflict between politics and religion has gained in importance substantially. Public interest usually focuses on the potential of conflict and violence of religion. However, the reciprocal relationship of politics and religion is much more complex than the prevailing media image of religiously motivated violence and Islamic terror conveys. Having neglected religion for quite a while, recent political science research demonstrates that there are many more facets to this topic. But at the same time there is also a substantial degree of uncertainty within the discipline how to deal analytically with the phenomenon of religion. This article intends to systemize the new and unclear state of research and thus to bring religion and its many intersections with politics into an analytical framework of political science. On the whole, the relationship of politics and religion proves to be one of the great overarching topics of a political science which intends to enlighten the options of democratic politics.

Antonius Liedhegener Politics and Religion in Today's Political Science. More than Political Violence in the Name of God 